\title{
Recursive SISO Impedance Modeling of Single- Phase Voltage Source Rectifiers
}

\author{
Jianheng Lin, Mei Su, Member, IEEE, Yao Sun, Member, IEEE, \\ Dongsheng Yang, Senior Member, IEEE, and Shiming Xie
}

\begin{abstract}
This paper proposes a recursive single-input singleoutput (SISO) impedance modeling framework for single-phase voltage source rectifiers, which can greatly simplify both the modeling procedure and the resulting impedance model. During the modeling process, frequency-coupling effects are modeled by the reduced-order transfer function vector rather than the transfer function matrix, so the modeling complexity is reduced. Moreover, the recursive SISO impedance model has an analytical form. And the recursive SISO impedance model can characterize the frequency-coupling dynamics of arbitrary order with a low computation burden. Experiments validate the accuracy of the recursive SISO impedance modeling.
\end{abstract}

Index Terms-Single-input single-output (SISO) impedance modeling, recursive function, single-phase voltage source rectifier.

\section{INTRODUCTION}

$\mathrm{O}$ VER the past decades, voltage source converters (VSCs) are being widely used in power grids, such as distributed generation, electric railway traction, and flexible power transmission [1]-[3]. With the increasing penetration of VSCs in power system, it not only improves efficiency and enhances control flexibility, but also leads to some stability issues introduced by the dynamic interactions between power converters and passive components (e.g., power filters and the grid impedance) [4], [5].

Many research efforts have been made to deal with VSC-grid interactions. For three-phase balanced VSC systems, the number of frequency couplings is typically bounded by two [4][6]. In order to incorporate the coupling dynamics, the threephase VSC is modeled as a $2 \times 2$ impedance matrix either in the $d q$ synchronous rotating frame [7] or in the $\alpha \beta$ stationary frame [8]. In $d q$ frame, the VSC system is linearized at constant DC operating points, leading to a linear-time-invariant (LTI) system. If performing linearization in the stationary frame, the resulting model is linear-time-periodic (LTP). Linearizing in different reference frames yields different types of impedance (i.e., $d q$ impedance, phasor impedance, and sequence impedance), and the correlation among different models are unveiled in [9] and [10]. On this basis, many detailed impedance models including different factors are formulated, e.g. constant power load [11] and PLL [12], [13]. Based on the complex vector notation [14] or algebraic calculation [15], the multiple-input multiple-output (MIMO) impedance matrix can be simplified into a single-input single-output (SISO) impedance model. This SISO impedance model not only facilitates the understanding of the consequence of impedance measurement under frequency couplings, but also provides an intuitive insight into the interactions among VSCs and power grids [16], [17].

However, in either three-phase unbalanced or single-phase systems, the time-periodic nature of the system introduces an infinite number of frequency couplings [4]. Instead of the impedance matrix with a dimension of two, the impedance matrix with infinite dimensions has to be used to model the terminal dynamics of these systems. Thus, the LTP frameworkbased modeling approaches are more feasible for the impedance modeling of such systems.

In general, there have been two general multi-frequency modeling methods capable of dealing with time-periodic systems. The generalized averaging modeling (GAM) was earlier introduced to capture the cross-coupling dynamics in [18] by S. R. Sanders et al., which is also known as dynamic phasor modeling [19], [20]. The key of GAM is to decompose the actual time-periodic waveform into the phasors in different frequency domains based on the harmonic balance principle. On the other hand, the harmonic-state-space (HSS) modeling method, which was firstly proposed by N. M. Wereley in [21], has been widely applied to address harmonic stability issues by J. B. Kwon et al. in [22], [23] and by V. Salis et al. in [24], [25]. Moreover, the counterpart of the HSS in the frequency domain is called as harmonic transfer function (HTF) approach [26], which directly derives the harmonic impedance in the frequency domain [2], [27], [28]. The core idea of HSS/HTF lies in the introduction of the exponential modulated periodic (EMP) signal, which maps the LTP model as an infinitedimensional LTI model. The models obtained from GAM or $\mathrm{HSS} / \mathrm{HTF}$ are characterized by MIMO with infinite dimensions, which brings about significant complexity to stability analysis and physical measurement.

In order to simplify the model, many efforts have been devoted to SISO equivalent impedance modeling method. Harmonic linearization, proposed by J. Sun et al. in [29], gives the describing-function-based model. However, the neglect of cross-coupling dynamics limits its accuracy, especially in the low-frequency regions [30]. In order to streamline and visualize the modeling process, the harmonic signal-flow graph method is proposed by S. Shah et al. in [31], [32]. The required convolution operations may increase the modeling difficulty as the considered harmonic order increases. In [33], Q. Qian et al. derives a single-frequency impedance model, incorporating the effect of the other two frequency-coupling components. This incorporation does improve the accuracy of the model, it is still inadequate to predict the impact of certain factors, such as the modulation method. The latest work in [34], presented by C. Zhang et al., extends the model to include coupling components 
of any order. However, the SISO impedance model is represented by a series of large-dimensional matrices, which degrades model analyticity and increases computation time.

Besides the model simplification, another obstacle is the modeling complexity resulting from the large-dimensional matrix operations. In the HTF model, each control loop of the VSC is modeled separately as a Toeplitz matrix with infinite dimensions, and then the models are combined based on their interconnections [26]-[28], [34]. In the modeling process, a series of matrix operations are required, especially the matrix inverse operation, which significantly increases the modeling complexity. Note that each row/column of the Toeplitz matrix can be obtained by frequency shifting another row/column. This observation implies that the information of the Toeplitz matrix is redundant in describing the frequency coupling effects.

In order to address the abovementioned challenges, a recursive SISO impedance modeling framework for singlephase voltage source rectifiers is proposed in this paper. The main contributions of the paper are summarized as:

1) A general and simple LTP modeling method is proposed by extending the LTI modeling. It does not require tedious convolution operations and large-dimensional matrix operations. Therefore, the modeling complexity is reduced.

2) The recursive SISO impedance model is analytical and easy to include harmonics of arbitrary order based on the required model accuracy. Compared with the HTF-based SISO model [34], the recursive SISO impedance model avoids complicated matrix inversion operations.

The rest of this paper is organized as follows. In Section II, a brief review of the basic concepts of the LTP modeling approach is reported. In Section III, the single-phase VSR system configuration is described. Section IV presents the modeling of harmonic admittance under no grid impedance. In Section V, the recursive SISO impedance model is derived and a detailed comparison with other existing models is given. In Section V, model validation is provided and the system stability analysis is conducted under different voltage loop control bandwidths. Finally, Section VI concludes this paper.

\section{FROM LTI MODELING TO LTP MODELING}

The information contained in the Toeplitz matrix is redundant, which increases the modeling complexity. In fact, one row/column of the Toeplitz matrix is enough to represent the complete system model. By utilizing this feature, the modeling complexity can be reduced. Based on this idea, a simple LTP modeling is introduced as follows.

\section{A. LTI Modeling Revisit}

To better understand the LTP modeling, a brief revisit of LTI modeling is given first. Consider a one-dimensional nonlinear time-invariant system

$$
\frac{d x}{d t}=f(x, u)
$$

where $x \in \mathbb{R}$ and $u \in \mathbb{R}$ represent a state variable and an input variable respectively, and $f$ is a nonlinear function from $\mathbb{R}^{2}$ to $\mathbb{R}$.

This system is autonomous, and its small-signal model can be derived as

$$
\frac{d \tilde{x}}{d t}=\left.\frac{\partial f}{\partial x}\right|_{\substack{x=\bar{x} \\ u=\bar{u}}} \cdot \tilde{x}+\left.\frac{\partial f}{\partial u}\right|_{\substack{x=\bar{x} \\ u=\bar{u}}} \cdot \tilde{u}:=\alpha \cdot \tilde{x}+\beta \cdot \tilde{u}
$$

where the superscripts ' $\sim$ ' and '-' represent the small-signal and steady-state quantities, respectively. Both $\alpha$ and $\beta$ are constant values determined by the steady-state operating points.

Applying Laplace transform to (2), it gives that

$$
s \cdot \tilde{x}(s)=\alpha \cdot \tilde{x}(s)+\beta \cdot \tilde{u}(s)
$$

where $s=j \omega$ and $\omega$ denotes the frequency variable. Then the transfer function from input to state is obtained as

$$
G(s)=\frac{\tilde{x}(s)}{\tilde{u}(s)}=\frac{\beta}{s-\alpha}
$$

By repeating the operation from (1) to (4), the small-signal model of a nonlinear time-invariant system in the frequency domain can be derived.

\section{B. LTP Modeling Approach}

The dynamics of a one-dimensional nonlinear time-periodic system is described as

$$
\frac{d x}{d t}=f(x(t), u(t))
$$

with its periodicity $T_{0}$ as

$$
\left\{\begin{array}{l}
x(t)=x\left(t+T_{0}\right) \\
u(t)=u\left(t+T_{0}\right)
\end{array}\right.
$$

The model of the single-phase grid-connected system is a typical example of this kind because of the steady-state timeperiodic operating trajectories.

The linearized model of such a nonautonomous system is

$$
\frac{d \tilde{x}}{d t}=\left.\frac{\partial f}{\partial x}\right|_{\begin{array}{c}
x(t)=\bar{x}(t) \\
u(t)=\bar{u}(t)
\end{array}} \cdot \tilde{x}+\left.\frac{\partial f}{\partial u}\right|_{\begin{array}{l}
x(t)=\bar{x}(t) \\
u(t)=\bar{u}(t)
\end{array}} \cdot \tilde{u}:=\alpha(t) \cdot \tilde{x}+\beta(t) \cdot \tilde{u}
$$

where $\alpha(t)$ and $\beta(t)$ are determined by the steady-state operating trajectories $\bar{x}(t)$ and $\bar{u}(t)$, satisfying

$$
\left\{\begin{array}{l}
\alpha(t)=\alpha\left(t+T_{0}\right) \\
\beta(t)=\beta\left(t+T_{0}\right)
\end{array}\right.
$$

As observed from (7), it is not able to derive the transfer function from the input to the state directly due to the timeperiodic coefficients.

From the Fourier transform theory, periodic signals satisfying Dirichlet conditions could be represented by a complex exponential series of infinite dimensions [35]. Thus, (8) can be rewritten by the Fourier series as

$$
\begin{aligned}
& \alpha(t)=\sum_{k=-\infty}^{\infty} a_{k} e^{j k \omega_{0} t}, \quad(k \in \mathbb{Z}) \\
& \beta(t)=\sum_{k=-\infty}^{\infty} b_{k} e^{j k \omega_{0} t}, \quad(k \in \mathbb{Z})
\end{aligned}
$$

where $\omega_{0}=2 \pi / T_{0}$. And $a_{k}$ and $b_{k}$ are complex Fourier coefficients, which are given by

$$
\begin{aligned}
& a_{k}=\frac{1}{T_{0}} \int_{0}^{T_{0}} \alpha(t) e^{-j k \omega_{0} t} d t \\
& b_{k}=\frac{1}{T_{0}} \int_{0}^{T_{0}} \beta(t) e^{-j k \omega_{0} t} d t
\end{aligned}
$$

Substituting (9) and (10) into (7) yields 


$$
\frac{d \tilde{x}}{d t}=\left[\sum_{k=-\infty}^{\infty} a_{k} e^{j k \omega_{0} t}\right] \cdot \tilde{x}+\left[\sum_{k=-\infty}^{\infty} b_{k} e^{j k \omega_{0} t}\right] \cdot \tilde{u}
$$

Then, according to the displacement property of the Laplace transform, the frequency domain expression of (13) is

$$
s \tilde{x}(s)=\left[\sum_{k=-\infty}^{\infty} a_{k} \tilde{x}\left(s-j k \omega_{0}\right)\right]+\left[\sum_{k=-\infty}^{\infty} b_{k} \tilde{u}\left(s-j k \omega_{0}\right)\right]
$$

It is found that $\tilde{x}$ at a given frequency $\omega$ is related to $\tilde{x}$ and $\tilde{u}$ not only at frequency $\omega$, but also multiple sideband frequencies $\omega \pm k \omega_{0}(k \in \mathbb{Z})$. This proves the existence of the frequencycoupling effect in the time-periodic systems.

Actually, (14) corresponds to one row of the Toeplitz matrix of the LTP system. Moreover, due to the arbitrariness of $s$, another row can be easily derived by frequency shifting as $\left(s-j m \omega_{0}\right) \tilde{x}\left(s-j m \omega_{0}\right)$

$=\left[\sum_{k=-\infty}^{\infty} a_{k} \tilde{x}\left(s-j(m+k) \omega_{0}\right)\right]+\left[\sum_{k=-\infty}^{\infty} b_{k} \tilde{u}\left(s-j(m+k) \omega_{0}\right)\right](15)$

By applying the frequency-shifting operation on (14) repeatedly, the frequency response from input to any order of harmonic can be constructed. The total dynamic propagations of the LTP system in the frequency domain are shown in Fig. 1.

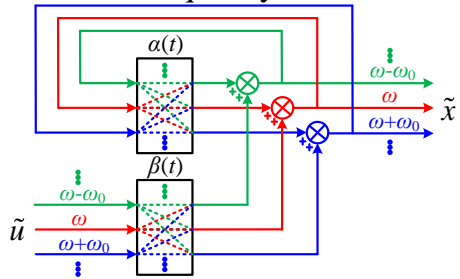

Fig. 1. Harmonic signal-flow graph of the time-periodic system.

In this way, the use of matrix operation can be avoided. Thus, the modeling complexity can be reduced. Also, the number of frequency-shifting operations required depends on the number of nonzero Fourier coefficients. In the practical analysis, truncation is usually required and the truncation order depends on the required accuracy [34]. By solving the obtained frequency-shifting equations, the SISO transfer function from $\tilde{u}(s)$ to $\tilde{x}(s)$ with consideration of frequency-coupling effects can be derived.

\section{SYSTEM CONFIGURATION}

The circuit configuration and control scheme of the studied single-phase voltage source rectifier (VSR) are depicted in Fig. 2. The main circuit is comprised of a power supply $u_{g}$, a grid impedance $L_{g} / R_{g}$, an input inductance $L_{f}$ in series with a resistance $R_{f}$, an H-bridge circuit, an output capacitance $C_{d c}$ and a load resistance $R_{d c}$. In the control diagram, a SOGI-PLL is used to obtain the phase $\theta$ of the point of common coupling (PCC) voltage $u_{i}$. And the DC-link voltage $u_{d c}$ and input current $i_{g}$ are regulated by a voltage loop and a current loop, respectively.

The studied system is split into two subsystems at PCC: one is the source subsystem and the other is the load subsystem, as shown in Fig. 3. The analysis of source and load systems can separately bring to an impedance-based representation for both of them. Based on the input impedance $Z(s)$ and the grid impedance $Z_{g}(s)$, the impedance-based stability criteria can be applied to determine the system stability.

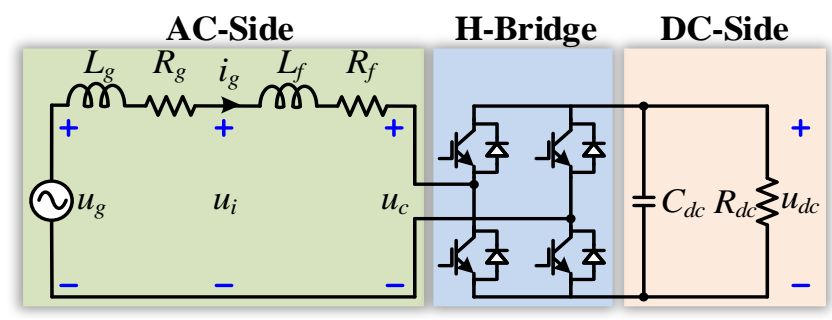

(a) Main circuit

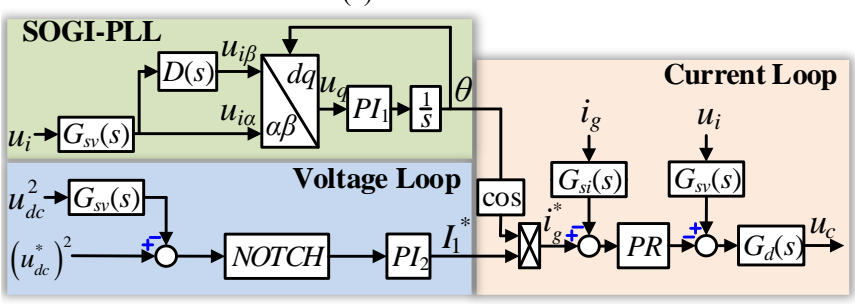

(b) Control diagram

Fig. 2. Schematic of a single-phase voltage source rectifier system.

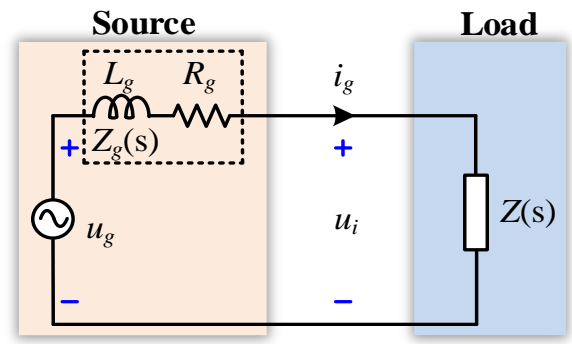

Fig. 3. Thevenin model of a single-phase voltage source rectifier.

In this system, the sampling delay is modeled as

$$
\begin{aligned}
G_{s i}(s) & =\frac{1}{1+s / \omega_{i}} \\
G_{s v}(s) & =\frac{1}{1+s / \omega_{v}}
\end{aligned}
$$

where $\omega_{i}$ and $\omega_{\nu}$ are the cutoff frequency of anti-aliasing filters for current and voltage measurement, respectively.

Besides, a second-order generalized integrator (SOGI), a notch filter (NOTCH), two proportional-integral (PI) regulators and a proportional-resonant (PR) regulator are given as

$$
\begin{gathered}
D(s)=\frac{2 \xi \omega_{1}^{2}}{s^{2}+2 \xi \omega_{1} s+\omega_{1}^{2}} \\
N O T C H(s)=\frac{s^{2}+\omega_{n}^{2}}{s^{2}+\sigma s+\omega_{n}^{2}} \\
P I_{m}(s)=k_{p m}+\frac{k_{i m}}{s} \quad(m=1,2) \\
P R(s)=k_{p c}+\frac{k_{r c} s}{s^{2}+\omega_{1}^{2}}
\end{gathered}
$$

where $\omega_{1}$ is the angular frequency of the grid, $\xi$ is the damping ratio, $\sigma$ is bandwidth coefficient, $\omega_{n}$ is the center frequency, $k_{p m}$ and $k_{i m}(m=1,2)$ are the PI controller parameters, and $k_{p c}$ and $k_{r c}$ are the PR controller parameters.

Zero-order hold $(\mathrm{ZOH})$ effects and calculation delays are taken into consideration as

$$
G_{d}(s)=e^{-s T_{s}} \frac{1-e^{-s T_{s}}}{s T_{s}}
$$

where $T_{s}$ is the control period. 


\section{HARMONIC AmitTANCE MODELING}

\section{A. SOGI-PLL Modeling}

The SOGI-PLL is used to track the phase $\theta$ of the PCC voltage $u_{i}$, which is used for generating current reference. The SOGI generates the $\pi / 4$-delay signal $u_{i \beta}$ of $u_{i \alpha}$. Then, applying the Park transformation to $u_{i \alpha}$ and $u_{i \beta}$, the $q$ axis voltage $u_{q}$ is obtained

$$
u_{q}(t)=-\sin [\theta(t)] u_{i \alpha}(t)+\cos [\theta(t)] u_{i \beta}(t)
$$

Perturbing the variables in the time-domain, the linearized form of (23) is derived as

$$
\tilde{u}_{q}(t)=-\sin \left(\omega_{1} t\right) \tilde{u}_{i \alpha}(t)+\cos \left(\omega_{1} t\right) \tilde{u}_{i \beta}(t)-V_{1} \tilde{\theta}(t)
$$

where $\omega_{1} t$ and $V_{1}$ are denoted as the phase and amplitude of the PCC voltage in steady-state, respectively.

According to (13) and (14), (24) can be rewritten as

$$
\begin{aligned}
\tilde{u}_{q}(s) & =\frac{j}{2}\left[\tilde{u}_{i \alpha}\left(s-j \omega_{1}\right)-\tilde{u}_{i \alpha}\left(s+j \omega_{1}\right)\right] \\
& +\frac{1}{2}\left[\tilde{u}_{i \beta}\left(s-j \omega_{1}\right)+\tilde{u}_{i \beta}\left(s+j \omega_{1}\right)\right]-V_{1} \tilde{\theta}(s)
\end{aligned}
$$

Based on the SOGI-PLL diagram, the following relations hold

$$
\begin{gathered}
\tilde{u}_{i \alpha}(s)=G_{s v}(s) \tilde{u}_{i}(s) \\
\tilde{u}_{i \beta}(s)=D(s) G_{s v}(s) \tilde{u}_{i}(s) \\
\tilde{\theta}(s)=H_{P L L}(s) \tilde{u}_{q}(s)
\end{gathered}
$$

where $H_{P L L}(s)=P I_{1}(s) / s$.

Substituting (26)-(28) into (25), it is deduced that

$$
\tilde{\theta}(s)=G_{P L L_{-} n}(s) \tilde{u}_{i}\left(s-j \omega_{1}\right)+G_{P L L_{-} p}(s) \tilde{u}_{i}\left(s+j \omega_{1}\right)
$$

where

$$
\begin{gathered}
G_{P L L_{-} n}(s)=G_{P L L}(s) G_{s v}\left(s-j \omega_{1}\right)\left[D\left(s-j \omega_{1}\right)+j\right] \\
G_{P L L_{-} p}(s)=G_{P L L}(s) G_{s v}\left(s+j \omega_{1}\right)\left[D\left(s+j \omega_{1}\right)-j\right] \\
G_{P L L}(s)=\frac{H_{P L L}(s)}{2\left[1+V_{1} H_{P L L}(s)\right]}
\end{gathered}
$$

Applying the frequency shift to (29) by $\pm k \omega_{1}(k \in \mathbb{Z})$, the harmonic signal-flow graph of the SOGI-PLL can be plotted as Fig. 4. It is observed that the perturbed phase $\tilde{\theta}$ at a given frequency $\omega$ is related to the PCC voltage $\tilde{u}_{i}$ at the two coupled frequencies $\omega \pm \omega_{1}$.

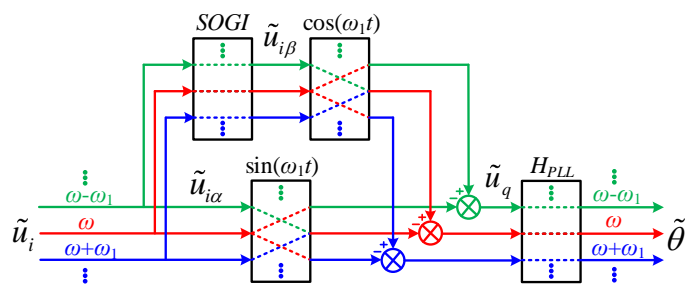

Fig. 4. Harmonic signal-flow graph of the SOGI-PLL.

\section{B. Voltage Loop Modeling}

Assume that the power losses on the switches of the rectifier are neglectable, the relation between the powers at ac side and dc side of Fig. 2(a) can be described as

$$
\frac{C_{d c}}{2} \frac{d u_{d c}^{2}(t)}{d t}+\frac{u_{d c}^{2}(t)}{R_{d c}}=u_{i}(t) i_{g}(t)-\frac{L_{f}}{2} \frac{d i_{g}^{2}(t)}{d t}-R_{f} i_{g}^{2}(t)
$$

Linearizing (33) gives (34) and its corresponding frequency domain expression is obtained as (35), shown at the bottom of the page. $I_{1}$ and $\varphi_{i 1}$ correspond to the magnitude and initial phase of the fundamental current in steady-state, respectively. And $G_{L}(s)$ is given by

$$
G_{L}(s)=\frac{R_{d c}}{C_{d c} R_{d c} s+2}
$$

From Fig. 2, the perturbed output of the voltage loop is

$$
\tilde{I}_{1}^{*}(s)=-H_{v}(s) \tilde{u}_{d c}^{2}(s)
$$

where

$$
H_{v}(s)=\mathrm{PI}_{2}(s) \operatorname{NOTCH}(s) G_{s v}(s)
$$

Based on the above calculations, the harmonic signal-flow graph of the voltage loop can be drawn as Fig. 5. In the voltage loop, $\tilde{I}_{1}^{*}$ at a given frequency $\omega$ is dependent on $\tilde{u}_{i}$ and $\tilde{i}_{g}$ at two coupled frequencies $\omega \pm \omega_{1}$.

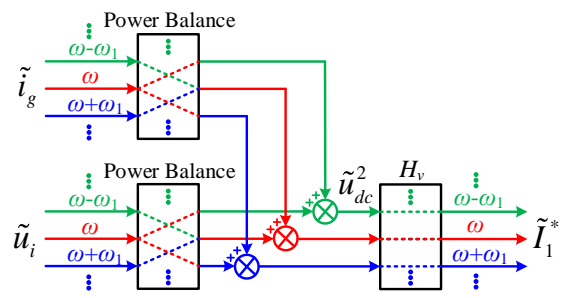

Fig. 5. Harmonic signal-flow graph of the voltage loop.

\section{Current Loop Modeling}

The grid current reference is

$$
i_{g}^{*}(t)=I_{1}^{*}(t) \cos [\theta(t)]
$$

The corresponding small-signal form of (39) is given as

$$
\tilde{i}_{g}^{*}(t)=-I_{1} \sin \left(\omega_{1} t\right) \tilde{\theta}(t)+\cos \left(\omega_{1} t\right) \tilde{I}_{1}^{*}(t)
$$

In the frequency domain, (40) is rewritten as

$$
\begin{aligned}
\tilde{i}_{g}^{*}(s) & =\frac{j I_{1}}{2}\left[\tilde{\theta}\left(s-j \omega_{1}\right)-\tilde{\theta}\left(s+j \omega_{1}\right)\right] \\
& +\frac{1}{2}\left[\tilde{I}_{1}^{*}\left(s-j \omega_{1}\right)+\tilde{I}_{1}^{*}\left(s+j \omega_{1}\right)\right]
\end{aligned}
$$

Due to the current regulator, the perturbed converter voltage including the feedforward term is

$\tilde{u}_{c}(s)=G_{d}(s)\left\{G_{s v}(s) \tilde{u}_{i}(s)-P R(s)\left[\tilde{i}_{g}^{*}(s)-G_{s i}(s) \tilde{i}_{g}(s)\right]\right\}$

Similarly, cross-coupling dynamics exist in the current loop that $\tilde{i}_{g}^{*}$ at a given frequency $\omega$ is coupled with $\tilde{\theta}$ and $\tilde{I}_{1}^{*}$ at two coupled frequencies $\omega \pm \omega_{1}$. And the harmonic signal-flow graph of the current loop is depicted as Fig. 6.

$$
\begin{aligned}
& \frac{C_{d c}}{2} \frac{d \tilde{u}_{d c}^{2}(t)}{d t}+\frac{\tilde{u}_{d c}^{2}(t)}{R_{d c}}=\left[V_{1} \cos \left(\omega_{1} t\right)+\omega_{1} L_{f} I_{1} \sin \left(\omega_{1} t+\varphi_{i 1}\right)\right] \tilde{i}_{g}(t)+I_{1} \cos \left(\omega_{1} t+\varphi_{i 1}\right)\left[\tilde{u}_{i}(t)-L_{f} \frac{d \tilde{i}_{g}(t)}{d t}-2 R_{f} \tilde{i}_{g}(t)\right] \\
& \tilde{u}_{d c}^{2}(s)=G_{L}(s)\left\{\begin{array}{l}
{\left[V_{1}-\left(s-j \omega_{1}\right) L_{f} I_{1} e^{j \varphi_{i 1}}-j \omega_{1} L_{f} I_{1} e^{j \varphi_{i 1}}-2 R_{f} I_{1} e^{j \varphi_{i 1}}\right] \tilde{i}_{g}\left(s-j \omega_{1}\right)+I_{1} e^{j \varphi_{i 1}} \tilde{u}_{i}\left(s-j \omega_{1}\right)} \\
+\left[V_{1}-\left(s+j \omega_{1}\right) L_{f} I_{1} e^{-j \varphi_{i 1}}+j \omega_{1} L_{f} I_{1} e^{-j \varphi_{i 1}}-2 R_{f} I_{1} e^{-j \varphi_{i 1}}\right] \tilde{i}_{g}\left(s+j \omega_{1}\right)+I_{1} e^{-j \varphi_{i 1}} \tilde{u}_{i}\left(s+j \omega_{1}\right)
\end{array}\right\}
\end{aligned}
$$




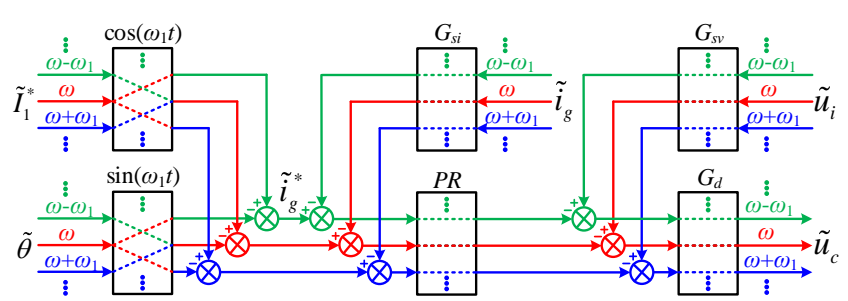

Fig. 6. Harmonic signal-flow graph of the current loop.

\section{Harmonic Admittance Modeling}

The harmonic signal-flow graphs show that the single-phase VSR system is a MIMO system essentially, which is different from the usual time-invariant system. According to the above analysis, it is worth noting that different signal-flow paths are translated copies of each other with frequency shifting proportional to the fundamental frequency $\omega_{1}$. Therefore, the system model can be extended to include harmonics of any order by frequency-shifting any one signal-flow path without complicated calculations.

The current dynamics in the average model is

$$
\left(L_{f} s+R_{f}\right) \tilde{i}_{g}(s)=\tilde{u}_{i}(s)-\tilde{u}_{c}(s)
$$

Substituting (29), (37), and (42) into (43), the dynamics between voltage and current at PCC is derived as

$G_{i,-2}(s) \tilde{i}_{g}\left(s-j 2 \omega_{1}\right)+G_{i, 0}(s) \tilde{i}_{g}(s)+G_{i, 2}(s) \tilde{i}_{g}\left(s+j 2 \omega_{1}\right)$

$=G_{u,-2}(s) \tilde{u}_{i}\left(s-j 2 \omega_{1}\right)+G_{u, 0}(s) \tilde{u}_{i}(s)+G_{u, 2}(s) \tilde{u}_{i}\left(s+j 2 \omega_{1}\right)$

where $G_{i,-2}(s), G_{i, 0}(s), G_{i, 2}(s), G_{u,-2}(s), G_{u, 0}(s)$, and $G_{u, 2}(s)$ are given in Appendix.

Neglecting the coupling terms in (44), the conventional input impedance is obtained as

$$
Z_{c}(s)=\frac{G_{i, 0}(s)}{G_{u, 0}(s)}
$$

In order to include the interactions among different frequency domains, frequency-shifting (44) to $s \pm j 2 \omega_{1}$ gives

$\tilde{i}_{g}\left(s-j 2 \omega_{1}\right)=\frac{G_{u, 2}\left(s-j 2 \omega_{1}\right) \tilde{u}_{i}(s)-G_{i, 2}\left(s-j 2 \omega_{1}\right) \tilde{i}_{g}(s)}{G_{i, 0}\left(s-j 2 \omega_{1}\right)}$

$\tilde{i}_{g}\left(s+j 2 \omega_{1}\right)=\frac{G_{u,-2}\left(s+j 2 \omega_{1}\right) \tilde{u}_{i}(s)-G_{i,-2}\left(s+j 2 \omega_{1}\right) \tilde{i}_{g}(s)}{G_{i, 0}\left(s+j 2 \omega_{1}\right)}$

where only the perturbed voltage term $\tilde{u}_{i}(s)$ is retained due to no grid impedance and the harmonic currents with quadruple line frequency deviation are assumed to be zero.

Substituting (46) and (47) into (44), the input impedance with capturing frequency coupling effects under no grid impedance is derived as (48), shown at the bottom of the page.

Substituting (48) into (46) and (47), the harmonic admittances are derived as

$$
\begin{aligned}
& Y_{n}(s)=\frac{\tilde{i}_{g}\left(s-j 2 \omega_{1}\right)}{\tilde{u}_{i}(s)}=\frac{G_{u, 2}\left(s-j 2 \omega_{1}\right)-G_{i, 2}\left(s-j 2 \omega_{1}\right) Y_{o p}(s)}{G_{i, 0}\left(s-j 2 \omega_{1}\right)} \\
& Y_{p}(s)=\frac{\tilde{i}_{g}\left(s+j 2 \omega_{1}\right)}{\tilde{u}_{i}(s)}=\frac{G_{u,-2}\left(s+j 2 \omega_{1}\right)-G_{i,-2}\left(s+j 2 \omega_{1}\right) Y_{o p}(s)}{G_{i, 0}\left(s+j 2 \omega_{1}\right)}
\end{aligned}
$$

The small-signal modeling illustrates that a perturbed PCC voltage $\tilde{u}_{i}$ at the frequency $\omega$ will generate three harmonic grid currents $\tilde{i}_{g}$ at the frequency $\omega$ and $\omega \pm \omega_{1}$, as depicted in Fig. 7. The existence of the FCE described by (49) and (50) poses a challenge to the modeling of the converter-grid interaction.

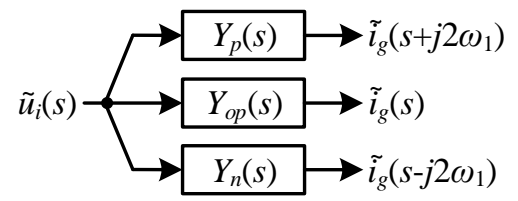

Fig. 7. Fundamental frequency coupling relation of the single-phase voltage source rectifier under no grid impedance.

\section{RECURSIVE SISO IMPEDANCE MODELING AND ANALYSIS}

When there is a grid impedance on the grid side, the harmonic currents would generate the voltage at the same frequency and thus constitute additional feedback loops. The multi-frequency diagram of a single-phase VSR with the additional closed loops is depicted as Fig. 8, where $s_{k}$ is defined as $s+j k \omega_{1}(k \in \mathbb{Z})$. The additional feedback loops are the translated copies of the fundamental loop (denoted by the black line) with frequency shifting $\pm k \omega_{1}$. Moreover, the order of harmonic would extend to infinite at the presence of the grid impedance.

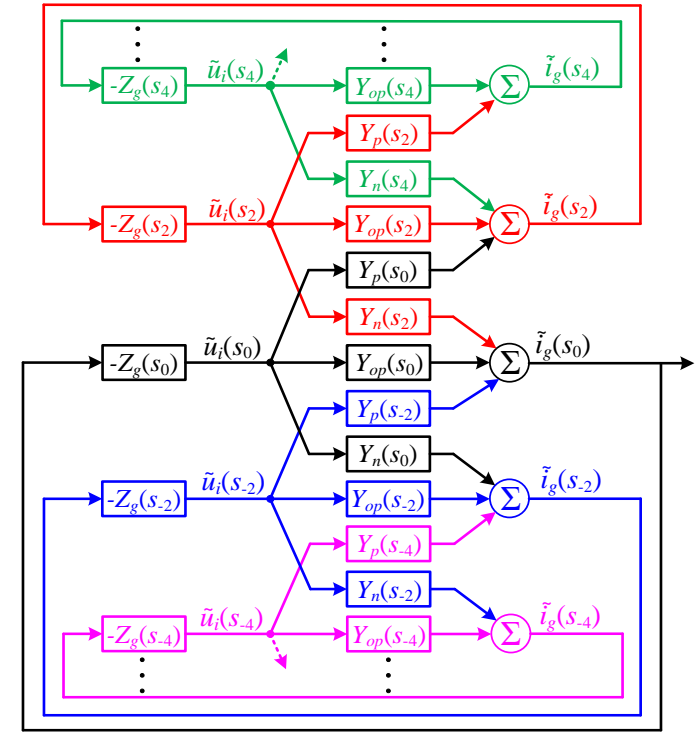

Fig. 8. Multi-frequency diagram of the single-phase voltage source rectifier considering the grid impedance.

\section{A. Recursive SISO Impedance Modeling}

According to Fig. 8, the relation between $\tilde{i}_{g}$ and $\tilde{u}_{i}$ in different frequency domains can be consistently established as

$$
\begin{aligned}
\tilde{i}_{g}\left(s_{K}\right) & =Y_{p}\left(s_{K-2}\right) \tilde{u}_{i}\left(s_{K-2}\right)+Y_{o p}\left(s_{K}\right) \tilde{u}_{i}\left(s_{K}\right) \\
& +Y_{n}\left(s_{K+2}\right) \tilde{u}_{i}\left(s_{K+2}\right)
\end{aligned}
$$

where $K \in \mathbb{Z}$. In addition, due to the existence of grid impedance, it is established that

$$
\tilde{i}_{g}\left(s_{K}\right)=-Y_{g}\left(s_{K}\right) \tilde{u}_{i}\left(s_{K}\right)
$$

where $K \in \mathbb{Z} \backslash 0$.

where $Y_{o p}(s)=1 / Z_{o p}(s)$.

$$
Z_{o p}(s)=\frac{G_{i, 0}(s)-G_{i, 2}\left(s-j 2 \omega_{1}\right) G_{i,-2}(s) / G_{i, 0}\left(s-j 2 \omega_{1}\right)-G_{i, 2}(s) G_{i,-2}\left(s+j 2 \omega_{1}\right) / G_{i, 0}\left(s+j 2 \omega_{1}\right)}{G_{u, 0}(s)-G_{i,-2}(s) G_{u, 2}\left(s-j 2 \omega_{1}\right) / G_{i, 0}\left(s-j 2 \omega_{1}\right)-G_{i, 2}(s) G_{u,-2}\left(s+j 2 \omega_{1}\right) / G_{i, 0}\left(s+j 2 \omega_{1}\right)}
$$




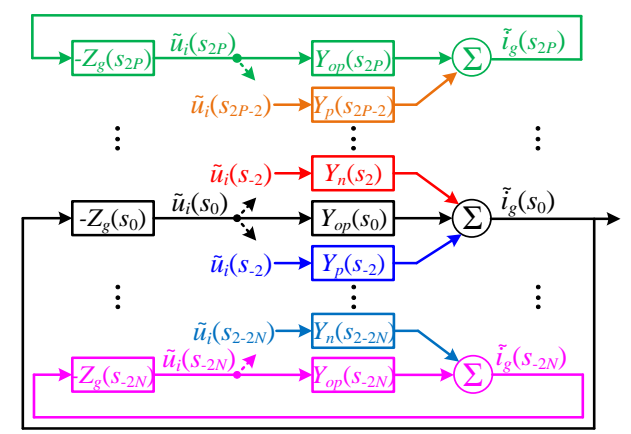

Fig. 9. Truncated multi-frequency diagram with $P$ positive loops and $N$ negative loops.

In order to obtain a model that can be used for analysis, truncation is required. Assume that the multi-loop system is truncated to $P(P \in \mathbb{N})$ positive loops and $N(N \in \mathbb{N})$ negative loops as depicted in Fig. 9, the truncation conditions are expressed as

$$
\begin{cases}\tilde{u}_{i}\left(s_{2 X}\right)=0, & X \geq P+1 \\ \tilde{u}_{i}\left(s_{-2 Y}\right)=0, & Y \geq N+1\end{cases}
$$

where $X \in \mathbb{N}$ and $Y \in \mathbb{N}$.

The positive loop truncation is taken as an example to illustrate how to obtain a truncated model. Based on the idea of mathematical induction, assume firstly that

$$
Y_{n}\left(s_{2 X}\right) \tilde{u}_{i}\left(s_{2 X}\right)=F_{P}(P-(X-1)) \tilde{u}_{i}\left(s_{2(X-1)}\right)
$$

where $1 \leq X \leq P+1, F_{P}(P-(X-1))$ is a mapping function from $\tilde{u}_{i}\left(s_{2(X-1)}\right)$ to $\tilde{u}_{i}\left(s_{2 X}\right)$ that needs to be determined, and $F_{P}(0)=0$ due to $(53)$.

Next, replacing $K$ in (51) with $2 X$ gives

$$
\begin{aligned}
\tilde{i}_{g}\left(s_{2 X}\right) & =Y_{p}\left(s_{2(X-1)}\right) \tilde{u}_{i}\left(s_{2(X-1)}\right)+Y_{o p}\left(s_{2 X}\right) \tilde{u}_{i}\left(s_{2 X}\right) \\
& +Y_{n}\left(s_{2(X+1)}\right) \tilde{u}_{i}\left(s_{2(X+1)}\right)
\end{aligned}
$$

Combining (52) and (54) into (55), it is obtained as

$$
\begin{aligned}
& Y_{n}\left(s_{2 X}\right) \tilde{u}_{i}\left(s_{2 X}\right) \\
& =\frac{-Y_{n}\left(s_{2 X}\right) Y_{p}\left(s_{2(X-1)}\right)}{Y_{g}\left(s_{2 X}\right)+Y_{o p}\left(s_{2 X}\right)+F_{P}(P-X)} \tilde{u}_{i}\left(s_{2(X-1)}\right)
\end{aligned}
$$

Comparing (54) and (56), $F_{P}(\bullet)$ can be solved as (57), shown at the bottom of the page.

In the same way, a recursive relationship for the negative loop truncation can be derived as

$$
Y_{p}\left(s_{-2 Y}\right) \tilde{u}_{i}\left(s_{-2 Y}\right)=F_{N}(N-(Y-1)) \tilde{u}_{i}\left(s_{-2(Y-1)}\right)
$$

where $1 \leq Y \leq N+1, F_{N}(\cdot)$ is given as (59), shown at the bottom of the page.

Finally, substituting $X=1$ into (54) and substituting $Y=1$ into (58) yield

$$
\begin{gathered}
Y_{n}\left(s_{2}\right) \tilde{u}_{i}\left(s_{2}\right)=F_{P}(P) \tilde{u}_{i}\left(s_{0}\right) \\
Y_{p}\left(s_{-2}\right) \tilde{u}_{i}\left(s_{-2}\right)=F_{N}(N) \tilde{u}_{i}\left(s_{0}\right)
\end{gathered}
$$

$$
\begin{aligned}
\tilde{i}_{g}\left(s_{0}\right) & =Y_{p}\left(s_{-2}\right) \tilde{u}_{i}\left(s_{-2}\right)+Y_{o p}\left(s_{0}\right) \tilde{u}_{i}\left(s_{0}\right) \\
& +Y_{n}\left(s_{2}\right) \tilde{u}_{i}\left(s_{2}\right)
\end{aligned}
$$

Combining (60)-(62), a recursive SISO impedance model can be derived as

$$
Z(s)=\frac{\tilde{u}_{i}\left(s_{0}\right)}{\tilde{i}_{g}\left(s_{0}\right)}=\frac{1}{Y_{o p}(s)+F_{N}(N)+F_{P}(P)}
$$

The recursive SISO impedance model has an analytical expression. Moreover, the recursive impedance is easy to incorporate harmonics of arbitrary order by only four transfer functions $\left(Y_{o p}(s), Y_{p}(s), Y_{n}(s)\right.$, and $\left.Y_{g}(s)\right)$ with their frequencyshifting copies. No matrix inversion operation as the HTF method [34] is required. When no grid impedance exists $\left(Z_{g}(s)\right.$ $=0$ ), it yields $F_{N}(\cdot)=0$ and $F_{P}(\cdot)=0$ such that the recursive impedance $Z(s)$ degrades to $Z_{o p}(s)$.

Overall, the recursive SISO impedance modeling method is summarized as Fig. 10, which includes two main steps. The first step is to establish the harmonic admittance model under no grid impedance condition, which can be deemed as the fundamental frequency coupling. The next step is to construct the complete system dynamics by frequency-shifting the fundamental frequency coupling, and then the recursive SISO impedance model can be derived based on the idea of mathematical induction.

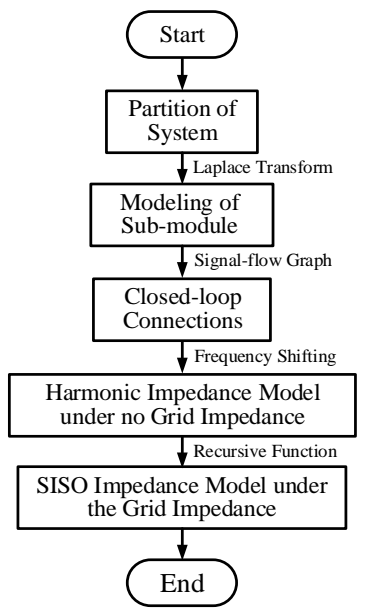

Fig. 10. Flowchart of the recursive impedance modeling method.

Remark: Considering a more general case, the relation between the voltage vector and the grid current vector in a $M$ phase system $\left(M \in \mathbb{N}^{+}\right)$can be expressed as

$$
\begin{aligned}
\boldsymbol{i}\left(s_{K}\right)= & \boldsymbol{Y}_{0}\left(s_{K}\right) \boldsymbol{u}\left(s_{K}\right)+\sum_{m=1}^{N_{1}} \boldsymbol{Y}_{p m}\left(s_{K-m}\right) \boldsymbol{u}\left(s_{K-m}\right) \\
& +\sum_{l=1}^{N_{2}} \boldsymbol{Y}_{\boldsymbol{n} l}\left(s_{K+l}\right) \boldsymbol{u}\left(s_{K+l}\right)
\end{aligned}
$$

where $\boldsymbol{i}\left(s_{K}\right)=\left[i_{1}\left(s_{K}\right), i_{2}\left(s_{K}\right), \ldots, i_{M}\left(s_{K}\right)\right]^{T}, \boldsymbol{u}\left(s_{K}\right)=\left[u_{1}\left(s_{K}\right), u_{2}\left(s_{K}\right), \ldots\right.$, $\left.u_{M}\left(s_{K}\right)\right]^{T}, N_{1} \in \mathbb{N}, N_{2} \in \mathbb{N}$, and $K \in \mathbb{Z}$. And $\boldsymbol{Y}_{p K}, \boldsymbol{Y}_{0}$, and $\boldsymbol{Y}_{p K}$ are all definite $M$-by- $M$ matrices.

Substituting $K=0$ into (51) gives

$$
\begin{gathered}
F_{P}(p)=\frac{-Y_{n}\left(s_{2(P-p+1)}\right) Y_{p}\left(s_{2(P-p)}\right)}{Y_{g}\left(s_{2(P-p+1)}\right)+Y_{o p}\left(s_{2(P-p+1)}\right)+F_{P}(p-1)} \text { with } F_{P}(0)=0 \\
F_{N}(n)=\frac{-Y_{p}\left(s_{-2(N-n+1)}\right) Y_{n}\left(s_{-2(N-n)}\right)}{Y_{g}\left(s_{-2(N-n+1)}\right)+Y_{o p}\left(s_{-2(N-n+1)}\right)+F_{N}(n-1)}
\end{gathered}
$$


In addition, the additional loop resulted from the grid admittance can be expressed as

$$
\boldsymbol{i}\left(s_{K}\right)=-\boldsymbol{Y}_{g}\left(s_{K}\right) \boldsymbol{u}\left(s_{K}\right)
$$

where $\boldsymbol{Y}_{g}\left(s_{K}\right)=\operatorname{diag}\left[Y_{g 1}\left(s_{K}\right), Y_{g_{2}}\left(s_{K}\right), \ldots, Y_{g M}\left(s_{K}\right)\right]$ and $K \in \mathbb{Z} \backslash 0$.

The truncation conditions are expressed as

$$
\begin{cases}\boldsymbol{u}\left(s_{X}\right)=0, & X \geq P+1 \\ \boldsymbol{u}\left(s_{-Y}\right)=0, & Y \geq N+1\end{cases}
$$

According to the idea of mathematical induction, two recursive relations can be derived as

$$
\begin{gathered}
\boldsymbol{u}\left(s_{K}\right)=\sum_{m=1}^{N_{1}} \boldsymbol{F}_{p m} \boldsymbol{u}\left(s_{K-m}\right) \\
\boldsymbol{u}\left(s_{-K}\right)=\sum_{l=1}^{N_{2}} \boldsymbol{F}_{n l} \boldsymbol{u}\left(s_{-K+l}\right)
\end{gathered}
$$

Combining (64), (67), and (68) and performing the frequencyshifting, the relation between $\boldsymbol{i}\left(s_{0}\right)$ and $\boldsymbol{u}\left(s_{0}\right)$ can be solved. Therefore, the developed recursive SISO modeling method has a great potential of being promoted to other time-periodic systems.

TABLE I

MAIN CIRCUIT AND CONTROL PARAMETERS

\begin{tabular}{cll}
\hline \hline Symbol & \multicolumn{1}{c}{ Description } & \multicolumn{1}{c}{ Value } \\
\hline$u_{g}$ & Grid voltage & $100 \mathrm{~V}(\mathrm{rms})$ \\
$\omega_{1}$ & Input angular frequency & $100 \pi \mathrm{rad} / \mathrm{s}$ \\
$L_{f}$ & Input filter inductance & $2.8 \mathrm{mH}$ \\
$R_{f}$ & Input filter resistance & $0.1 \Omega$ \\
$C_{d c}$ & Output capacitance & $240 \mu \mathrm{F}$ \\
$R_{d c}$ & Load resistance & $62.5 \Omega$ \\
$\omega_{i} / \omega_{v}$ & ADC cutoff frequency & $10^{4} \pi \mathrm{rad} / \mathrm{s}$ \\
$\xi$ & Damping ratio of the SOGI & 0.707 \\
$k_{p 1}$ & Proportional gain of the PLL & 6.3 \\
$k_{i 1}$ & Integral gain of the PLL & 7896 \\
$k_{p 2}$ & Proportional gain of the voltage regulator & $2.8 \times 10^{-5}$ \\
$k_{i 2}$ & Integral gain of the voltage regulator & 0.03 \\
$k_{p c}$ & Proportional gain of the current regulator & 6.7 \\
$k_{r c}$ & Resonant gain of the current regulator & 11640 \\
$\omega_{n}$ & Center frequency of Notch filter & $200 \pi \mathrm{rad} / \mathrm{s}$ \\
$\sigma$ & Bandwidth coefficient of Notch filter & 4737 \\
$u_{d c}^{*}$ & Output voltage reference & $250 \mathrm{~V}$ \\
$T_{s}$ & Control period & $50 \mu \mathrm{s}$ \\
\hline
\end{tabular}

B. Frequency Response Analysis of Recursive Impedance

The model accuracy depends on the chosen truncation order. When finite truncation is performed, frequency response deviation may occur, as shown in Fig. 11 (main parameters are listed in TABLE I). As observed, the selection of truncation order mainly influences the frequency response of $Z(s)$ around the fundamental frequency. And the frequency response deviation decreases as the truncation order increases. The evaluation of truncation order has also been discussed in the literature [34], but an explicit formula has not yet been provided. Thus, the truncation parameters $P$ and $N$ are usually evaluated iteratively. In the work presented in the following, $N=3$ and $P$ $=3$ have been chosen in the following work.

In order to better visualize the influence of grid impedance, the impedance model $Z(s)$ with different grid impedances are plotted in Fig. 12. $Z_{\mathrm{X}}(s)(\mathrm{X}=\mathrm{A}, \mathrm{B}, \mathrm{C}, \mathrm{D})$ are used to represent $Z(s)$ under different grid impedances $\left(Z_{\mathrm{A}}(s): L_{g}=0 \mathrm{mH}, R_{g}=0\right.$ $\Omega ; Z_{\mathrm{B}}(s): L_{g}=1.5 \mathrm{mH}, R_{g}=1 \Omega ; Z_{\mathrm{C}}(s): L_{g}=3.5 \mathrm{mH}, R_{g}=1 \Omega$; $\left.Z_{\mathrm{D}}(s): L_{g}=5.5 \mathrm{mH}, R_{g}=1 \Omega\right)$. From Fig. 12 , it is observed that evident discrepancies occur around the fundamental frequency as the grid impedance changes, which shows that the rectifier impedance depends on the grid impedance. There exist two valley points in the low-frequency regions, whose frequencies are symmetric about the fundamental frequency. And, with the increase of the grid impedance, the magnitude gain of the rectifier impedance decreases, implying that the ability to suppress voltage disturbances near the valley frequencies degrades.

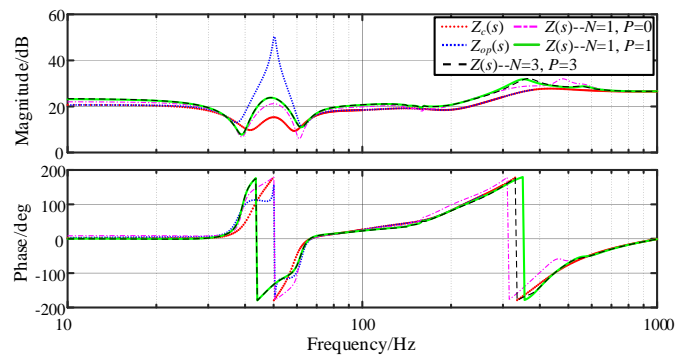

Fig. 11. Comparative analysis of SISO impedance with different truncation orders under $L_{g}=5.5 \mathrm{mH}$ and $R_{g}=1.5 \Omega$.

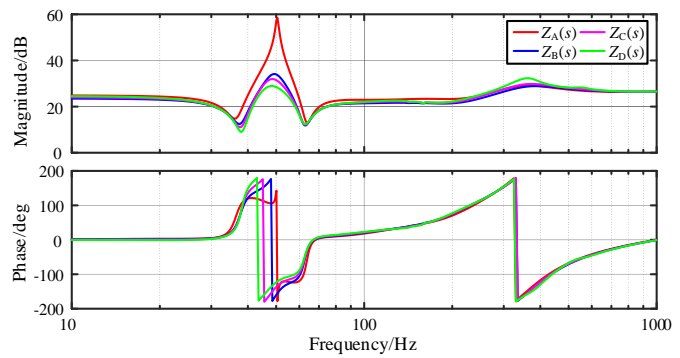

Fig. 12. Comparative analysis of SISO impedance with different grid impedances. $Z_{\mathrm{A}}(s): L_{g}=0 \mathrm{mH}$ and $R_{g}=0 \Omega ; Z_{\mathrm{B}}(s): L_{g}=1.5 \mathrm{mH}$ and $R_{g}=1 \Omega$; $Z_{\mathrm{C}}(s): L_{g}=3.5 \mathrm{mH}$ and $R_{g}=1 \Omega ; Z_{\mathrm{D}}(s): L_{g}=5.5 \mathrm{mH}$ and $R_{g}=1 \Omega$.

Based on the Matlab platform, the computation time required to calculate the impedance response of the HTF matrix-based model [34] and the proposed recursive SISO model at one thousand frequency points is shown in Fig. 13. As seen, the computation time required by the recursive SISO model is always less than that of the HTF matrix-based model, verifying the superiority of the recursive SISO model in the computational burden. In fact, the reason for the superiority lies in the elimination of large-dimensional matrix operations. The recursive SISO model can save approximately $16.7 \%$ time when the truncation order is 1 . Moreover, the polynomial fitting curve shows that the computation complexity of the HTF matrix-based model will increases exponentially as the truncation order increases. In contrast, the computation complexity of the recursive SISO model increases almost linearly as the truncation order increases.

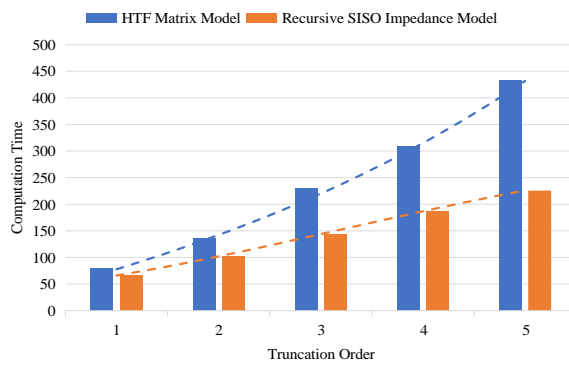

Fig. 13. Computation time comparisons of $Z_{H T F}(s)$ and $Z(s)$ with different truncation order. 
TABLE II

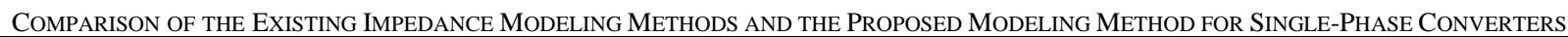

\begin{tabular}{|c|c|c|c|c|c|}
\hline & $\begin{array}{l}\text { Harmonic linearization } \\
\text { method }^{[29]}\end{array}$ & $\begin{array}{l}\text { Harmonic signal flow } \\
\text { graph method }^{[30]-[31]}\end{array}$ & $\begin{array}{l}\text { Multi-frequency } \\
\operatorname{method}^{[33]}\end{array}$ & $\begin{array}{l}\text { Harmonic transfer } \\
\text { function method }{ }^{[34]}\end{array}$ & Proposed method \\
\hline Modeling object & Diode Rectifier & PFC Rectifier & PFC Inverter & PFC Rectifier & PFC Rectifier \\
\hline $\begin{array}{l}\text { Frequency- } \\
\text { coupling } \\
\text { components }\end{array}$ & Diode bridge & $\begin{array}{c}\text { PLL } \\
\text { DC-link voltage }\end{array}$ & PLL & $\begin{array}{c}\text { PLL } \\
\text { DC-link voltage }\end{array}$ & $\begin{array}{c}\text { PLL } \\
\text { DC-link voltage }\end{array}$ \\
\hline $\begin{array}{c}\text { Submodule } \\
\text { modeling }\end{array}$ & Describing function & Describing function & $\begin{array}{c}\text { Transfer function } \\
\text { vector }\end{array}$ & $\begin{array}{c}\text { Transfer function } \\
\text { matrix }\end{array}$ & $\begin{array}{c}\text { Transfer function } \\
\text { vector }\end{array}$ \\
\hline $\begin{array}{l}\text { Coupling } \\
\text { frequencies }\end{array}$ & Arbitrary & $\omega \pm \omega_{1}$ & $\begin{array}{c}\omega \\
\omega \pm 2 \omega_{1}\end{array}$ & Arbitrary & Arbitrary \\
\hline $\begin{array}{l}\text { Grid impedance } \\
\text { effect }\end{array}$ & Ignored & Ignored & Considered & Considered & Considered \\
\hline $\begin{array}{c}\text { Steady-state DC } \\
\text { voltage trajectory }\end{array}$ & All & $\mathrm{DC}$ & l & All & All \\
\hline Model analyticity & Good & Good & Good & Medium & Good \\
\hline
\end{tabular}

\section{Comparison Between the Existing Impedance Models and the Proposed Recursive Impedance Model}

Table II summarizes the comparison results of the existing impedance modeling methods and the proposed modeling method for the single-phase converters. As shown in Table II, the modeling methods can be mainly categorized into two types: non-linearization-based methods and linearization-based methods.

The harmonic linearization modeling method and the harmonic signal-flow graph modeling method can be directly applied to the nonlinear time-periodic systems without linearization. However, the cross-coupling dynamics caused by the grid impedance are neglected in the modeling process. The multi-frequency modeling method, the harmonic transfer function modeling method, and the proposed modeling method all directly linearize the nonlinear time-periodic system around ac steady-state trajectories. The multi-frequency modeling only considers a limited number of frequency couplings, which may be a potential limitation for model accuracy. The use of largedimensional matrices degrades model analyticity and increases the computation effort of the harmonic transfer function model. The proposed modeling method gives an analytical and compact model, which can include the harmonics of arbitrary order without a large computation burden. Thus, it is a promising choice for single-phase converter modeling.

\section{EXPERIMENTAL VALIDATION}

In this section, a prototype of the single-phase voltage source rectifier is built to verify the correctness of the proposed models, as shown in Fig. 14. The system specifications are listed in TABLE I. The control platform is based on a floating-point Digital Signal Processor (DSP) TMS320F28335. The grid voltage and the injected small perturbation from $10 \mathrm{~Hz}$ to $1 \mathrm{kHz}$ are generated by a Regenerative Grid Simulator (Chroma 61830). And the measured signals are sent to the Frequency Response Analyzer Bode 100.

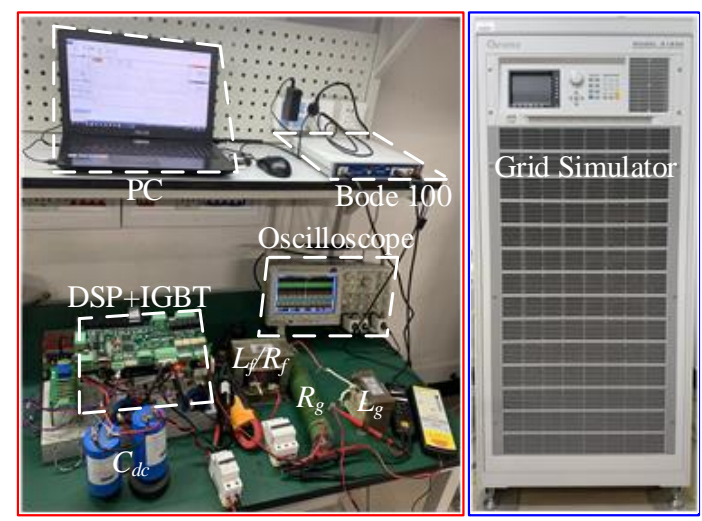

Fig. 14. Experimental setup for SISO impedance measurement verification.

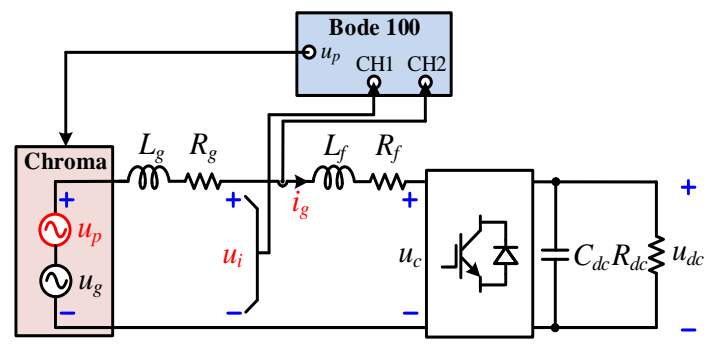

Fig. 15. Impedance measurement scheme for single-phase converters.

\section{A. Frequency Scan Validation}

The implementation of the impedance measurement scheme is depicted in Fig. 15. A small-signal perturbation voltage $u_{p}$ is injected, which excites the perturbations of corresponding frequency on the PCC voltage and input current. The PCC voltage signal $u_{i}$ sends to the $\mathrm{CH} 1$ port, and the grid current signal $i_{g}$ transmits to the $\mathrm{CH} 2$ port. The measured impedance is obtained by plotting the Bode diagram of $\mathrm{CH} 1 / \mathrm{CH} 2$.

The frequency response characteristics of the built impedance models and its corresponding measured results under different grid impedances are plotted as shown in Fig. 16. As shown, the measured results are in good accordance with the calculated impedance $Z(s)$, which validates the accuracy of the proposed impedance modeling approach. The conventional impedance $Z_{c}(s)$, which only considers the fundamental perturbed components, has a large deviation in the regions 
around the fundamental frequency. The $Z_{o p}(s)$ achieves higher accuracy compared with $Z_{c}(s)$, but the accuracy will degrade as the grid impedance increases. These cases show that the couplings between the grid impedance and the rectifier play an important role on the model accuracy. Thus, it is suggested that the interactions resulted from the grid impedance should be considered in the input impedance modeling of the single-phase voltage source rectifier system.

\section{B. Stability Analysis}

The Right-Half-Plane (RHP) zeros of $1+Z(s) / Z_{g}(s)$ are analyzed for determining the system stability [28], [36]. Fig. 17 shows the stability analysis under a fixed grid impedance $\left(L_{g}=\right.$ $5.5 \mathrm{mH}, R_{g}=1 \Omega$ ) when the voltage loop bandwidth $f_{b}$ is configured as $19 \mathrm{~Hz}$ and $21 \mathrm{~Hz}$, respectively. From Fig. 17(a), only Left-Half-Plane (LHP) zeros are found when $f_{b}=19 \mathrm{~Hz}$, indicating a stable system. While for $f_{b}=21 \mathrm{~Hz}$ as shown in Fig. 17(b), the RHP zeros are found at $35 \mathrm{~Hz}$ and $65 \mathrm{~Hz}$, meaning that the system is unstable.

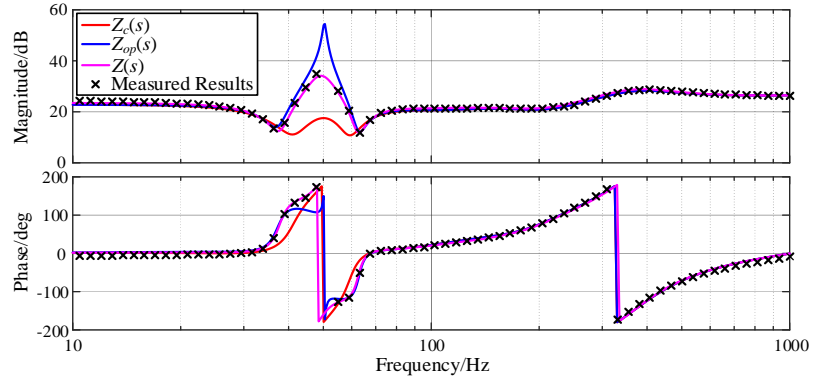

(a) Case A: $\left(L_{g}, R_{g}\right)=(1.5 \mathrm{mH}, 1 \Omega)$

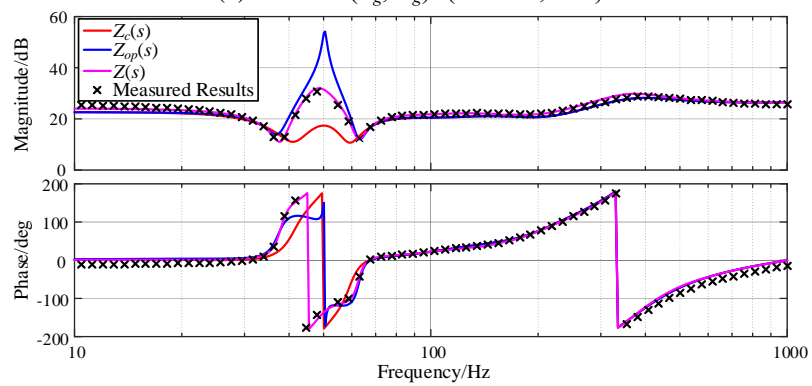

(b) Case B: $\left(L_{g}, R_{g}\right)=(3.5 \mathrm{mH}, 1 \Omega)$

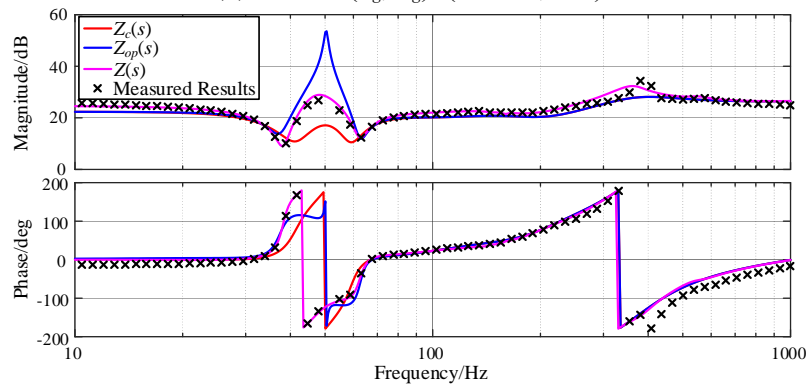

(c) Case C: $\left(L_{g}, R_{g}\right)=(5.5 \mathrm{mH}, 1 \Omega)$

Fig. 16. Impedance responses. Solid lines: Model prediction; Dots: Experimental results.

Experiments are carried out with $f_{b}=19 \mathrm{~Hz}$ and $f_{b}=21 \mathrm{~Hz}$ in Fig. 18 and Fig. 19, respectively. It is seen that for $f_{b}=19 \mathrm{~Hz}$, the system operates steadily. Whereas for $f_{b}=21 \mathrm{~Hz}$, lowfrequency oscillations can be found in the DC voltage $u_{d c}$ and the grid current $i_{g}$. By analyzing the spectrum of $i_{g}$, the oscillation frequencies of $35.5 \mathrm{~Hz}$ and $65.5 \mathrm{~Hz}$ are all identified as shown in Fig. 19(b), which closely agrees with the analyzed results in Fig. 17.

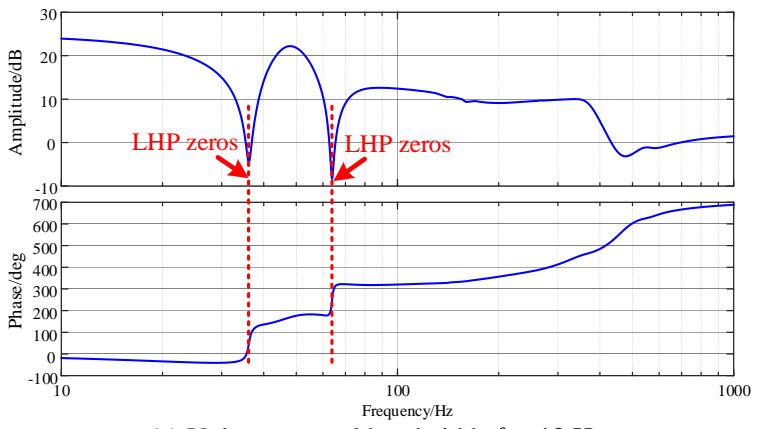

(a) Voltage control bandwidth $f_{b}=19 \mathrm{~Hz}$

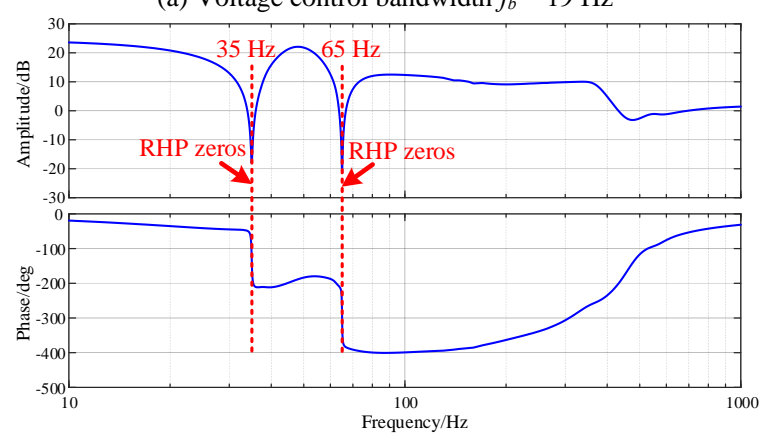

(b) Voltage control bandwidth $f_{b}=21 \mathrm{~Hz}$

Fig. 17. Bode diagrams of $1+Z(s) / Z_{g}(s)$.

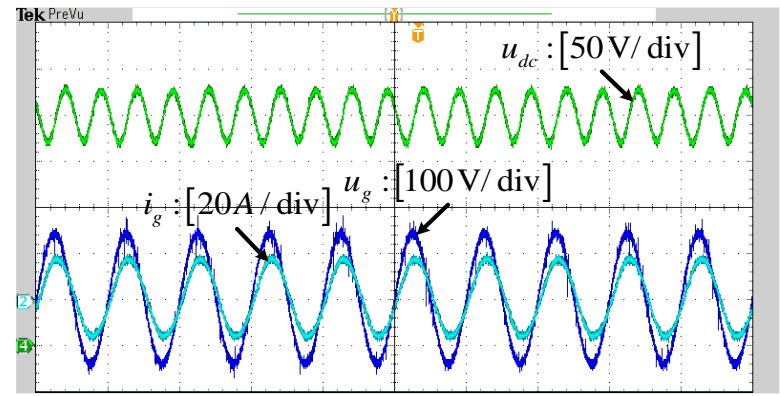

Fig. 18. Experimental waveforms with $f_{b}=19 \mathrm{~Hz}$.

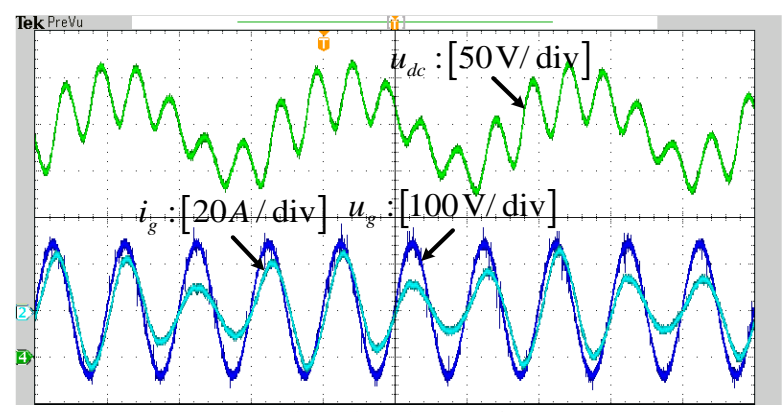

(a) Time-domain waveforms

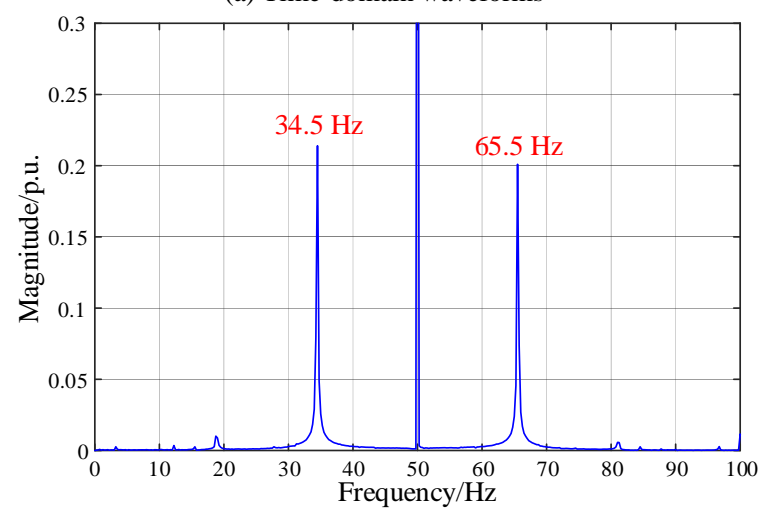

(b) Spectrum of $i_{g}$

Fig. 19. Experimental waveforms with $f_{b}=21 \mathrm{~Hz}$. 
The stability analysis indicates that the proposed impedance model is accurate for stability prediction. Moreover, it shows that a lower bandwidth $f_{b}$ of the voltage loop can achieve better stability in terms of the VSC-grid interaction. Increasing voltage loop bandwidth $f_{b}$ will move two LHP zeros to the RHP, and thus the low-frequency oscillation occurs.

\section{CONCLUSION}

For the single-phase converters, the LTI modeling method is no longer suitable because of the ac steady-state trajectories. In this work, a recursive SISO impedance modeling framework for single-phase VSR is presented by extending the LTI modeling method. Compared with the existing modeling methods, the recursive SISO impedance modeling method has the following advantages:

1) The frequency-coupling dynamics are modeled by the reduced-order transfer function vector, which avoids the convolution operations and large-dimensional matrix operations. Therefore, the modeling complexity is reduced.

2) The elimination of matrix inversion operation in the resulting recursive SISO impedance model greatly improves the model analyticity and reduces the computational complexity. The calculation time required by the recursive SISO impedance model is always less than that of the HTF matrix-based model [34].

\section{APPENDIX}

The transfer function of the harmonic admittance model

$$
\begin{gathered}
G_{i,-2}(s)=-\frac{1}{2} G_{d}(s) P R(s) G_{i n}(s) \\
G_{i, 0}(s)=L_{f} s+R_{f}+G_{d}(s) P R(s) G_{s i}(s) \\
-\frac{G_{d}(s) P R(s)}{2}\left[G_{i p}\left(s-j 2 \omega_{1}\right)+G_{i n}\left(s+j 2 \omega_{1}\right)\right] \\
G_{i, 2}(s)=-\frac{1}{2} G_{d}(s) P R(s) G_{i p}(s) \\
G_{u,-2}(s)=\frac{G_{d}(s) P R(s)}{2}\left[G_{u n}(s)+j I_{1} G_{P L L_{-} n}\left(s-j \omega_{1}\right)\right] \\
G_{u, 0}(s)=1-G_{d}(s) G_{s v}(s) \\
+\frac{j I_{1} G_{d}(s) P R(s)}{2}\left[G_{P L L_{-} p}\left(s-j \omega_{1}\right)-G_{P L L_{-} n}\left(s+j \omega_{1}\right)\right] \\
+\frac{G_{d}(s) P R(s)}{2}\left[G_{u p}\left(s-j 2 \omega_{1}\right)+G_{u n}\left(s+j 2 \omega_{1}\right)\right] \\
G_{u, 2}(s)=\frac{1}{2} G_{d}(s) P R(s)\left[G_{u p}(s)-j I_{1} G_{P L L_{-} p}\left(s+j \omega_{1}\right)\right] \\
G_{u n}(s)=-I_{1} e^{j \varphi_{i 1}} G_{v o l}\left(s-j \omega_{1}\right) \\
G_{u p}(s)=-I_{1} e^{-j \varphi_{i 1}} G_{v o l}\left(s+j \omega_{1}\right) \\
G_{i n}(s)=-G_{v o l}\left(s-j \omega_{1}\right)\left[\begin{array}{l}
V_{1}-\left(s-j 2 \omega_{1}\right) L_{f} I_{1} e^{j \varphi_{i 1}} \\
-j \omega_{1} L_{f} I_{1} e^{j \varphi_{i 1}}-2 R_{f} I_{1} e^{j \varphi_{i 1}}
\end{array}\right] \\
{\left[\begin{array}{l}
V_{1}-\left(s+j 2 \omega_{1}\right) L_{f} I_{1} e^{-j \varphi_{i 1}} \\
+j \omega_{1} L_{f} I_{1} e^{-j \varphi_{i 1}}-2 R_{f} I_{1} e^{-j \varphi_{i 1}}
\end{array}\right]}
\end{gathered}
$$

$$
G_{v o l}(s)=H_{v}(s) G_{L}(s)
$$

\section{REFERENCES}

[1] F. Blaabjerg, R. Teodorescu, M. Liserre, and A. V. Timbus, "Overview of control and grid synchronization for distributed power generation systems," IEEE Trans. Ind. Electron., vol. 53, no. 5, pp. 1398-1409, Oct. 2006.

[2] E. Mollerstedt and B. Bernhardsson, "Out of control because of harmonics-an analysis of the harmonic response of an inverter locomotive," IEEE Control Syst. Mag., vol. 20, no. 4, pp. 70-81, Aug. 2000.

[3] L. Zhang, L. Harnefors and H.-P. Nee, "Interconnection of two very weak AC systems by VSC-HVDC links using power-synchronization control," IEEE Trans. Power Systems, vol. 26, no. 1, pp. 344-355, Feb. 2011.

[4] X. Wang and F. Blaabjerg, "Harmonic stability in power electronicbased power systems: concept, modeling, and analysis," IEEE Trans. Smart Grid, vol. 10, no. 3, pp. 2858-2870, May 2019.

[5] D. Yang, X. Wang, F. Liu, K. Xin, Y. Liu, and F. Blaabjerg, "Symmetrical PLL for SISO impedance modeling and enhanced stability in weak grids," IEEE Trans. Power Electron., vol. 35, no. 2, pp. 14731483, Feb. 2020.

[6] A. Rygg, M. Molinas, C. Zhang, and X. Cai, "A modified sequencedomain impedance definition and its equivalence to the dq-domain impedance definition for the stability analysis of AC power electronic system," IEEE J. Emerg. Sel. Topics Power Electron., vol. 4, no. 4, pp. 1383-1396, Dec. 2016.

[7] L. Harnefors, M. Bongiorno and S. Lundberg, "Input-admittance calculation and shaping for controlled voltage-source converters," IEEE Trans. Ind. Electron., vol. 54, no. 6, pp. 3323-3334, Dec. 2007.

[8] M. K. Bakhshizadeh, X. Wang, F. Blaabjerg, J. Hjerrild, L. Kocewiak, C. L. Bak and B. Hesselbak, "Couplings in phase domain impedance modeling of grid-connected converters," IEEE Trans. Power Electron., vol. 31, no. 10, pp. 6792-6796, Oct. 2016.

[9] S. Shah and L. Parsa, "Impedance modeling of three-phase voltage source converters in dq, sequence, and phasor domains," IEEE Trans. Energ. Conv., vol. 32, no. 3, pp. 1139-1150, Sep. 2017.

[10] X. Wang, L. Harnefors and F. Blaabjerg, "Unified impedance model of grid-connected voltage-source converters," IEEE Trans. Power Electron., vol. 33, no. 2, pp. 1775-1787, Feb. 2018.

[11] M. Belkhayat, "Stability criteria for AC power systems with regulated loads," Ph.D. dissertation, Purdue Univ., West Lafayette, IN, Dec. 1997.

[12] L. Harnefors, M. Bongiorno and S. Lundberg, "Input-admittance calculation and shaping for controlled voltage-source converters," IEEE Trans. Ind. Electron., vol. 54, no. 6, pp. 3323-3334, Dec. 2007.

[13] B. Wen, D. Boroyevich, R. Burgos, P. Mattavelli and Z. Shen, "Analysis of d-q small-signal impedance of grid-tied inverters," IEEE Trans. Power Electron., vol. 31, no. 1, pp. 675-687, Jan. 2016.

[14] L. Harnefors, "Modeling of three-phase dynamic systems using complex transfer functions and transfer matrices," IEEE Trans. Ind. Electron., vol. 54, no. 4, pp. 2239-2248, Aug. 2007.

[15] C. Zhang, X. Cai, A. Rygg, and M. Molinas, "Sequence domain SISO equivalent models of a grid-tied voltage source converter system for small-signal stability analysis," IEEE Trans. Energ. Conv., vol. 33, no. 2, pp. 741-749, Jun. 2018.

[16] J. Liu, X. Du, Y. Shi, and H.-M. Tai, "Impedance measurement of threephase inverter in the stationary frame using frequency response analyzer," IEEE Trans. Power Electron., vol. 35, no. 9, pp. 9390-9401, Sep. 2020.

[17] D. Yang and X. Wang, "Unified modular state-space modeling of gridconnected voltage-source converters," IEEE Trans. Power Electron., vol. 35, no. 9, pp. 9700-9715, Sep. 2020.

[18] S. R. Sanders, J. M. Noworolski, X. Z. Liu and G. C. Verghese, "Generalized averaging method for power conversion circuits," IEEE Trans. Power Electron., vol. 6, no. 2, pp. 251-259, Apr. 1991.

[19] A. M. Stankovic, S. R. Sanders and G. C. Verghese, "Dynamic phasors in modeling and analysis of unbalanced polyphaser ac machines," IEEE Trans. Energy Conv., vol. 17, no. 1, pp. 107-113, Mar. 2002.

[20] S. Lissandron, L. Santa, P. Mattavelli and B. Wen, "Experimental validation for impedance-based small-signal stability analysis of singlephase interconnected power systems with grid-feeding inverters," IEEE J. Emerg. Sel. Topics Power Electron., vol. 4, no. 1, pp. 103-115, Mar. 2016. 
[21] N. M. Wereley, “Analysis and control of linear periodically time varying systems," Ph.D. dissertation, Dept. of Aeronautics and Astronautics, MIT Inst. Technol., 1991.

[22] J. B. Kwon, X. Wang, F. Blaabjerg, C. L. Bak, A. R. Wood, and N. R. Watson, "Harmonic instability analysis of a single-phase grid-connected converter using a harmonic state-space modeling method," IEEE Trans. Ind. Appl., vol. 52, no. 5, pp. 4188-4200, Sep. 2016.

[23] J. B. Kwon, X. Wang, F. Blaabjerg, C. L. Bak, V. S. Sularea and C. Busca "Harmonic interaction analysis in a grid-connected converter using harmonic state-space (HSS) modeling," IEEE Trans. Power Appl., vol. 32, no. 9, pp. 6823-6835, Sep. 2017.

[24] V. Salis, A. Costabeber, S. M. Cox, P. Zanchetta and A. Formentini, "Stability boundary analysis in single-phase grid-connected inverters with PLL by LTP theory," IEEE Trans. Power Electron., vol. 33, no. 5, pp. 4023-4036, May. 2018.

[25] V. Salis, A. Costabeber, S. M. Cox and P. Zanchetta, "Stability assessment of power-converter-based ac systems by LTP theory: eigenvalue analysis and harmonic impedance estimation," IEEE $J$. Emerg. Sel. Topics Power Electron., vol. 5, no. 4, pp. 1513-1525, Dec. 2017.

[26] E. Mollerstedt, "Dynamic analysis of harmonics in electrical systems," Ph.D. dissertation, Dept. Automatic Control, Lund Inst. Technol., Lund, Sweden, 2000.

[27] C. Zhang, M. Molinas, A. Rygg, J. Lyu and X. Cai, "Harmonic transferfunction-based impedance modeling of a three-phase VSC for asymmetric AC grids stability analysis," IEEE Trans. Power Electron., vol. 34, no.12, pp. 12552-12566, Dec. 2019.

[28] Y. Liao, X. Wang, X. Yue, and L. Harnefors, "Complex-valued multifrequency admittance model of three-phase VSCs in unbalanced grids," IEEE J. Emerg. Sel. Topics Power Electron., vol. 8, no.12, pp. 1934-1946, Jun. 2020.

[29] J. Sun and K. Karimi, "Small-signal input impedance modeling of linefrequency rectifiers," IEEE Trans. Aerosp. Electron. Syst., vol. 44, no. 4, pp. 1489-1497, Oct. 2008.

[30] H. Zhang, Z. Liu, S. Wu, and Z. Li, "Input impedance modeling and verification of single-phase voltage source converters based on harmonic linearization," IEEE Trans. Power Electron., vol. 34, no. 9, pp. 85448554, Sep. 2019.

[31] S. Shah and L. Parsa, "On impedance modeling of single-phase voltage source converters," IEEE Energy Conv. Cong. and Expo. (ECCE), pp. 1$8,2016$.

[32] S. Shah, P. Koralewicz, V. Gevorgian, and L. Parsa, "Small-signal modeling and design of phase-locked loops using harmonic signal-flow graphs," IEEE Trans. Energ. Conv., vol. 35, no. 2, pp. 600-610, Jun. 2020.

[33] Q. Qian, S. Xie, J. Xu, K. Xu, S. Bian and N. Zhong, "Output impedance modeling of single-phase grid-tied inverters with capturing the frequency-coupling effect of PLL," IEEE Trans. Power Electron., vol. 35, no. 5, pp. 5479-5495, May 2020.

[34] C. Zhang, M. Molinas, S. Foyen, J. A. Suul and T. Isobe, "Harmonic domain SISO equivalent impedance modeling and stability analysis of a single-phase grid connected VSC," IEEE Trans. Power Electron., vol. 35, no. 9, pp. 9770-9783, Sep. 2020.

[35] J. Borwein, D. Bailey and R. Girgensohn, Experimentation in Mathematics: Computational Paths to Discovery. Massachusetts, A K Peters. 2004.

[36] Y. Liao and X. Wang, "Impedance-based stability analysis for interconnected converter systems with open-loop RHP poles," IEEE Trans. Power Electron., vol. 35, no. 4, pp. 4388-4397, Apr. 2020. 